\title{
Open-label trial and randomized, double-blind, placebo-controlled, crossover trial of hydrogen- enriched water for mitochondrial and inflammatory myopathies
}

Mikako Ito ${ }^{1+}$, Tohru $\mathrm{lbi}^{2+}$, Ko Sahashi $^{3}$, Masashi Ichihara ${ }^{4}$, Masafumi Ito ${ }^{5}$ and Kinji Ohno ${ }^{*^{*}}$

\begin{abstract}
Background: Molecular hydrogen has prominent effects on more than 30 animal models especially of oxidative stress-mediated diseases and inflammatory diseases. In addition, hydrogen effects on humans have been reported in diabetes mellitus type 2, hemodialysis, metabolic syndrome, radiotherapy for liver cancer, and brain stem infarction. Hydrogen effects are ascribed to specific radical-scavenging activities that eliminate hydroxyl radical and peroxynitrite, and also to signal-modulating activities, but the detailed molecular mechanisms still remain elusive. Hydrogen is a safe molecule that is largely produced by intestinal bacteria in rodents and humans, and no adverse effects have been documented.

Methods: We performed open-label trial of drinking 1.0 liter per day of hydrogen-enriched water for 12 weeks in five patients with progressive muscular dystrophy (PMD), four patients with polymyositis/dermatomyositis (PM/DM), and five patients with mitochondrial myopathies (MM), and measured 18 serum parameters as well as urinary 8isoprostane every 4 weeks. We next conducted randomized, double-blind, placebo-controlled, crossover trial of 0.5 liter per day of hydrogen-enriched water or placebo water for 8 weeks in 10 patients with DM and 12 patients with $\mathrm{MM}$, and measured 18 serum parameters every 4 weeks.

Results: In the open-label trial, no objective improvement or worsening of clinical symptoms was observed. We, however, observed significant effects in lactate-to-pyruvate ratios in PMD and MM, fasting blood glucose in PMD, serum matrix metalloproteinase-3 (MMP3) in PM/DM, and serum triglycerides in PM/DM. In the double-blind trial, no objective clinical effects were observed, but a significant improvement was detected in lactate in MM. Lactateto-pyruvate ratios in MM and MMP3 in DM also exhibited favorable responses but without statistical significance. No adverse effect was observed in either trial except for hypoglycemic episodes in an insulin-treated MELAS patient, which subsided by reducing the insulin dose.
\end{abstract}

Conclusions: Hydrogen-enriched water improves mitochondrial dysfunction in MM and inflammatory processes in PM/DM. Less prominent effects with the double-blind trial compared to the open-label trial were likely due to a lower amount of administered hydrogen and a shorter observation period, which implies a threshold effect or a dose-response effect of hydrogen.

\footnotetext{
* Correspondence: ohnok@med.nagoya-u.ac.jp

† Contributed equally

${ }^{1}$ Division of Neurogenetics, Center for Neurological Diseases and Cancer, Nagoya University Graduate School of Medicine, 65 Tsurumai, Showa-ku,

Nagoya 466-8550, Japan

Full list of author information is available at the end of the article
} 


\section{Background}

Ohsawa and colleagues first reported an effect of hydrogen gas on cerebral infarction in June 2007 [1]. Effects of hydrogen administered in the forms of inhaled gas, drinking water, instillation, and intraperitoneal injection have been reported for 31,4 , and 5 diseases in animal models, cells, and humans, respectively [2]. Hydrogen exhibits prominent effects especially on oxidative stressmediated diseases and inflammatory diseases in rodents. Hydrogen scavenges hydroxyl radicals and less efficiently peroxynitrite [1]. The radical-scavenging activities, however, are unlikely to be an exclusive mechanism, because the amount of radical oxygen species generated in rodents and humans is much more than the amount of hydrogen molecules taken up by the body. Indeed, the amount of hydrogen taken up by drinking hydrogenenriched water (HEW) is 100 or more times less than that by inhaling $2 \%$ hydrogen gas, but drinking HEW exhibits beneficial effects as good as or even better than inhaling $2 \%$ hydrogen gas in rodents [2-4], which suggests the lack of a simple dose-response effect. Our previous study on type 1 allergy also indicates that hydrogen suppresses type 1 allergy by acting as a gaseous signal modulator not as a radical scavenger [5].

Effects of hydrogen in humans have been examined in five studies. First, a randomized, double-blind, placebocontrolled crossover study of $900 \mathrm{ml} /$ day of HEW for 8 weeks in 30 patients with diabetes mellitus type 2 demonstrated significant decreases of electronegative charge-modified LDL cholesterol, small dense LDL, and urinary 8-isoprostanes [6]. Second, an open-label trial of electrolyzed hydrogen-enriched hemodialysis solution in 9 patients for 4 months [7] and 21 patients for 6 months [8] showed significant decreases of systolic blood pressure before and after dialysis, as well as of plasma monocyte chemoattractant protein 1 and myeloperoxidase. Third, an open-label trial of 1.5-2.0 liters per day of HEW for 8 weeks in 20 subjects with metabolic syndrome exhibited a $39 \%$ increase of urinary superoxide dismutase (SOD), a $43 \%$ decrease of urinary thiobarbituric acid reactive substances (TBARS), an $8 \%$ increase of high density lipoprotein (HDL)-cholesterol, and a $13 \%$ decrease of total cholesterol/HDL-cholesterol ratio [9]. Fourth, a randomized placebo-controlled study of 1.5-2.2 liters/day of HEW for 6 weeks in 49 patients receiving radiotherapy for malignant liver tumors showed marked improvements of QOL scores [10]. As the study was not blinded, subjective QOL scores tended to be overestimated by a placebo effect, but objective markers for oxidative stress were also significantly decreased. Fifth, drip infusion of hydrogenenriched saline in combination with Edaravone, a clinically approved radical scavenger for cerebral infarction, for 7 days in 8 patients with brain stem infarction was compared to 24 such patients receiving Edaravone alone [11]. Although the study was not randomized and not blinded, MRI markers of patients infused with hydrogen showed significant improvements and accelerated normalization.

Being prompted by the prominent effects of hydrogen on inflammatory diseases and oxidative stress-mediated diseases especially in rodents, we performed an openlabel trial of drinking 1.0 liter per day of HEW for 12 weeks in 14 patients with muscle diseases, and identified improvement in four parameters: (i) a decrease of the lactate-to-pyruvate ratio in mitochondrial myopathies (MM) and progressive muscular dystrophy (PMD); (ii) a decrease of serum matrix metalloproteinase-3 (MMP3) in polymyositis/dermatomyositis (PM/DM), (iii) a decrease of fasting glucose in PMD, and (iv) a decrease of serum triglycerides in PM/DM. We then conducted a randomized, double-blind, placebo-controlled, crossover trial of 0.5 liter per day of HEW for 8 weeks in $12 \mathrm{MM}$ and $10 \mathrm{DM}$ cases. We observed that HEW significantly improved serum lactate in MM. In both studies, some patients reported subjective improvement of fatigability, diarrhea, and myalgia, but others reported floating sensation and worsening of diarrhea. We observed no objective improvement or worsening of clinical symptoms during each study. Our studies imply that HEW improves clinical parameters in $\mathrm{MM}$ and $\mathrm{PM} / \mathrm{DM}$, but 0.5 liter/day for 8 weeks is likely to be insufficient to demonstrate statistically significant effects.

\section{Patients and methods Patients}

For the open-label trial, we recruited 5 patients with PMD, 4 patients with $\mathrm{PM} / \mathrm{DM}$, and 5 patients with MM. The PMD patients comprised 1 male with Miyoshi myopathy and 4 females with limb girdle muscular dystrophy type $2 \mathrm{~B}$ with an average age and $\mathrm{SD}$ of $50.4 \pm$ 15.9 years (range 25 - 66). The PM/DM patients comprised 2 males and 2 females with an average age of $53.8 \pm 24.8$ years (range $29-83$ ). All the PM/DM cases were taking 5 - $10 \mathrm{mg}$ of prednisolone per day and were well controlled. The MM patients comprised 4 cases with MELAS (2 males and 2 females with an average age of $45.8 \pm 12.3$ years, range $37-64$ ) and a 54-yearold female with chronic progressive external ophthalmoplegia (CPEO).

For the randomized, double-blind, placebo-controlled, crossover trial, we recruited 12 patients with $\mathrm{MM}$ and 10 patients with DM. The MM patients comprised 5 cases with MELAS ( 2 males and 3 females with an average age of $44.6 \pm 17.6$ years, range $20-65$ ), as well as 7 cases with CPEO ( 3 males and 4 females with an average age of $49.1 \pm 11.1$ years, range $29-61$ ). The DM patients comprised 3 males and 7 females with an 
average age of $49.6 \pm 13.7$ years (range $32-66$ ). All the DM patients were well controlled with $5-10 \mathrm{mg}$ prednisolone per day. Three MM and three DM patients participated in both trials. Both trials were approved by the Ethical Review Board of the Aichi Medical University. Informed consent was obtained from each patient.

\section{Protocols}

We purchased $500 \mathrm{ml}$ HEW or placebo water in aluminum pouch from Blue Mercury Inc. (Tokyo, Japan). We measured hydrogen concentrations using an $\mathrm{H} 2-\mathrm{N}$ hydrogen needle sensor attached to a PA2000 2-Channel Picoammeter (Unisense Science, Aarhus, Denmark). The hydrogen concentrations were $\sim 0.5 \mathrm{ppm}$ ( $\sim 31 \%$ saturation). We also confirmed that hydrogen in placebo water was undetectable with our system. For each trial, we instructed patients to evacuate the air from the pouch and to close a plastic cap tightly every time after they drink water to keep the hydrogen concentration as high as possible.

For the open-label trial, patients took 1.0 liter per day of HEW in five to ten divided doses for 12 weeks. We measured 18 serum and one urinary parameters and recorded clinical symptoms at $0,4,8,12,16$ weeks.

For the double-blind trial, patients took 0.5 liter per day of HEW or placebo water in two to five divided doses for 8 weeks. Between the 8-week trials with HEW and placebo, we placed a 4 -week washout period. We measured 18 serum parameters and recorded clinical symptoms at $0,4,8,12,16,20,24$ weeks. In the doubleblind trial, we did not measure urinary 8 -isoprostane levels.

The data were statistically analyzed using one-way repeated measures ANOVA for the open-label trial and two-way repeated measures ANOVA for the doubleblind trial, both followed by the Bonferroni's multiple comparison test using Prism version 4.0c (Graphpad Software, San Diego, CA).

\section{Results}

\section{Open-label trial}

Fourteen patients with PMD, PM/DM, and MM participated in the study and no patient dropped out of the study. Patients took 1.0 liter of HEW for 12 weeks and we measured 18 serum and one urinary parameters every 4 weeks (Table 1 ). We observed no objective improvement or worsening of clinical symptoms during the study. All the patients reported increased micturition frequency. Two MELAS patients reported improvement of fatigability, and another MELAS patient complained mild occasional floating sensation. We estimated statistical significance using one-way repeated measures ANOVA analysis and detected five parameters (Figure 1). Serum lactate-to-pyruvate $(\mathrm{L} / \mathrm{P})$ ratios of MM patients were high before the study, and were decreased during the study (Figure 1A). Serum L/P ratios and fasting glucose levels of PMD patients were elevated after the study, but the values were still within normal ranges (Figures 1B and 1C). Serum MMP3 levels of DM patients were decreased down to $72.9 \%$ of those before HEW, which were again increased after the study (Figure 1D). Serum triglyceride levels of DM patients were elevated after the study (Figure 1E).

\section{Randomized, double-blind, placebo-controlled, crossover trial}

Twelve MM and ten DM patients participated in the study and no patient dropped out of the study. Patients took 0.5 liter of HEW or placebo water for 8 weeks and we measured 18 serum parameters every 4 weeks (Table 2). An MM patient reported increased micturition frequency on HEW. A DM patient reported subjective improvement of fatigability and diarrhea on HEW, but an MM patient rather complained increased diarrhea at first on HEW. Another DM patient reported an improvement of myalgia on HEW. A MELAS patient had hypoglycemic episodes only on HEW, but the episodes subsided after the insulin dose was decreased. We observed no objective improvement or worsening of clinical symptoms during the study. Two-way repeated measures ANOVA analysis revealed that only serum lactate levels were significantly decreased in MM by HEW (Figure 2A). Temporal profiles of serum L/P ratios in MM (Figure 2B) and of serum MMP3 levels in DM (Figure 2C) also demonstrated favorable responses to HEW but without statistical significance.

\section{Discussion}

We performed open-label and double-blind studies of HEW on myopathic patients. In the open-label study, we observed statistical significance of hydrogen effects in four parameters: L/P ratios in MM and PMD; fasting glucose in PMD; MMP3 in PM/DM; and triglycerides in $\mathrm{PM} / \mathrm{DM}$ (Figure 1). In the double-blind study, serum lactate levels were significantly improved in MM. L/P ratios in MM and MMP3 in DM were also improved but without statistical significance (Figure 2). Small numbers of participants in both the open-label and double-blind studies might have failed to disclose statistically significant effects of HEW.

In $\mathrm{MM}$, the mitochondrial electron transfer system (mETS) is compromised by mutations in mitochondrial DNA [12]. This results in a decreased influx of NADH into mETS and elevates NADH levels in the cytoplasm, which facilitates conversion of pyruvate to lactate by lactate dehydrogenase. Thus, lactate and $\mathrm{L} / \mathrm{P}$ ratio are useful surrogate markers to estimate functions of mETS, and are usually abnormally elevated in MM [12]. Defective mETS also causes leakage of electrons from 
Table 1 Open-label trial of HEW in 14 myopathic patients

\begin{tabular}{|c|c|c|c|c|c|c|c|c|c|}
\hline & \multicolumn{3}{|c|}{ Progressive muscular dystrophy (PMD) } & \multicolumn{3}{|c|}{ Polymyositis (PM)/Dermatomyositis (DM) } & \multicolumn{3}{|c|}{ Mitochondrial myopathies (MM) } \\
\hline & Before & 12 weeks & After & Before & 12 weeks & After & Before & 12 weeks & After \\
\hline CK (U/L) & $3067 \pm 1492$ & $3419 \pm 1610$ & $3107 \pm 2382$ & $124 \pm 31$ & $180 \pm 97$ & $140 \pm 86$ & $187 \pm 75$ & $124 \pm 47$ & $156 \pm 40$ \\
\hline $\mathrm{HbA1c}(\%)$ & $5.25 \pm 0.44$ & $5.14 \pm 0.31$ & $5.16 \pm 0.42$ & $6.68 \pm 1.61$ & $6.70 \pm 1.53$ & $6.90 \pm 2.03$ & $7.40 \pm 1.70$ & $7.32 \pm 1.48$ & $7.38 \pm 1.74$ \\
\hline Fasting glucose (mmol/L) & $5.52 \pm 0.16^{* *}$ & $5.51 \pm 0.08^{* *}$ & $5.82 \pm 0.11^{* *}$ & $7.66 \pm 0.11$ & $7.29 \pm 1.57$ & $7.69 \pm 1.85$ & $8.94 \pm 3.24$ & $9.31 \pm 4.18$ & $8.96 \pm 3.19$ \\
\hline $\begin{array}{c}\text { Lactate } \\
(\mathrm{mmol} / \mathrm{L})\end{array}$ & $0.95 \pm 0.34$ & $1.15 \pm 0.40$ & $1.35 \pm 0.40$ & $1.42 \pm 0.18$ & $1.66 \pm 0.32$ & $1.30 \pm 0.27$ & $1.84 \pm 0.50$ & $1.87 \pm 0.78$ & $1.73 \pm 0.65$ \\
\hline $\begin{array}{c}\mathrm{L} / \mathrm{P} \\
\text { ratio }\end{array}$ & $12.1 \pm 0.7^{*}$ & $10.7 \pm 1.3^{*}$ & $13.6 \pm 2.2^{*}$ & $13.1 \pm 0.9$ & $15.0 \pm 3.2$ & $12.7 \pm 1.0$ & $20.7 \pm 2.9^{*}$ & $14.9 \pm 3.5^{*}$ & $20.3 \pm 3.1^{*}$ \\
\hline Creatinine $(\mu \mathrm{mol} / \mathrm{L})$ & $34.8 \pm 3.1$ & $34.5 \pm 6.9$ & $34.7 \pm 8.8$ & $58.6 \pm 13.7$ & $56.3 \pm 10.7$ & $56.6 \pm 14.1$ & $48.8 \pm 9.0$ & $47.7 \pm 9.7$ & $48.6 \pm 8.8$ \\
\hline BUN (mmol/L) & $4.74 \pm 1.16$ & $4.20 \pm 0.60$ & $4.21 \pm 1.05$ & $4.33 \pm 0.71$ & $4.11 \pm 0.48$ & $4.68 \pm 0.74$ & $5.28 \pm 1.69$ & $5.89 \pm 1.09$ & $5.00 \pm 1.58$ \\
\hline Uric acid ( $\mu \mathrm{mol} / \mathrm{dL})$ & $295 \pm 46$ & $315 \pm 61$ & $300 \pm 30$ & $319 \pm 44$ & $331 \pm 71$ & $329 \pm 40$ & $208 \pm 50$ & $220 \pm 60$ & $217 \pm 45$ \\
\hline $\begin{array}{l}\text { Urinary 8-isoprostane } \\
\text { (ng/mg Cr) }\end{array}$ & $303 \pm 155$ & $392 \pm 173$ & n.d. & $222 \pm 88$ & $237 \pm 86$ & n.d. & $274 \pm 117$ & $261 \pm 59$ & n.d. \\
\hline T-chol (mmol/L) & $5.42 \pm 0.99$ & $5.74 \pm 1.02$ & $5.70 \pm 0.81$ & $5.28 \pm 0.31$ & $5.55 \pm 0.93$ & $5.97 \pm 1.35$ & $4.52 \pm 0.75$ & $4.53 \pm 0.34$ & $4.42 \pm 0.77$ \\
\hline LDL-chol (mmol/L) & $3.30 \pm 1.05$ & $2.86 \pm 1.46$ & $2.99 \pm 1.15$ & $2.66 \pm 0.28$ & $3.21 \pm 0.99$ & $3.13 \pm 1.02$ & $2.44 \pm 0.43$ & $2.27 \pm 0.44$ & $2.22 \pm 0.45$ \\
\hline HDL-chol (mmol/L) & $1.62 \pm 0.21$ & $1.49 \pm 0.19$ & $1.62 \pm 0.18$ & $2.01 \pm 0.73$ & $1.99 \pm 0.76$ & $2.02 \pm 0.67$ & $1.14 \pm 0.79$ & $1.03 \pm 0.72$ & $1.04 \pm 0.69$ \\
\hline Triglycerides (mmol/L) & $1.31 \pm 0.46$ & $3.62 \pm 4.83$ & $3.07 \pm 3.67$ & $2.17 \pm 0.63^{*}$ & $2.01 \pm 1.21^{*}$ & $3.09 \pm 1.22^{*}$ & $0.78 \pm 0.34$ & $0.89 \pm 0.45$ & $0.73 \pm 0.37$ \\
\hline WBC $\left(10^{9} / L\right)$ & $5.30 \pm 1.32$ & $5.40 \pm 1.12$ & $4.80 \pm 0.38$ & $10.78 \pm 2.08$ & $8.35 \pm 3.41$ & $10.10 \pm 0.17$ & $5.12 \pm 1.27$ & $7.27 \pm 2.29$ & $5.93 \pm 1.35$ \\
\hline $\operatorname{RBC}\left(10^{12} / \mathrm{L}\right)$ & $4.17 \pm 0.64$ & $3.79 \pm 0.42$ & $3.87 \pm 0.80$ & $4.01 \pm 0.62$ & $4.34 \pm 0.36$ & $4.49 \pm 0.45$ & $4.33 \pm 0.43$ & $4.31 \pm 0.78$ & $4.35 \pm 0.59$ \\
\hline Platelets $\left(10^{9} / \mathrm{L}\right)$ & $262 \pm 42$ & $260 \pm 33$ & $270 \pm 20$ & $337 \pm 123$ & $265 \pm 82$ & $270 \pm 65$ & $215 \pm 25$ & $207 \pm 28$ & $217 \pm 25$ \\
\hline Hematocrit & $0.375 \pm 0.040$ & $0.374 \pm 0.038$ & $0.407 \pm 0.055$ & $0.337 \pm 0.069$ & $0.376 \pm 0.041$ & $0.395 \pm 0.045$ & $0.381 \pm 0.036$ & $0.370 \pm 0.047$ & $0.378 \pm 0.038$ \\
\hline MMP3 (ng/ml) & n.d. & n.d. & n.d. & $307.8 \pm 59.1^{*}$ & $224.3 \pm 53.4^{*}$ & $283.3 \pm 77.6^{*}$ & n.d. & n.d. & n.d. \\
\hline $\operatorname{lgG}(\mathrm{mg} / \mathrm{dl})$ & n.d. & n.d. & n.d. & $1343 \pm 470$ & $1396 \pm 550$ & $1429 \pm 581$ & n.d. & n.d. & n.d. \\
\hline
\end{tabular}

Values represent mean \pm SD. n.d., not determined. ${ }^{*} p<0.05$ and ${ }^{* *} p<0.005$ by one-way repeated measures ANOVA. 


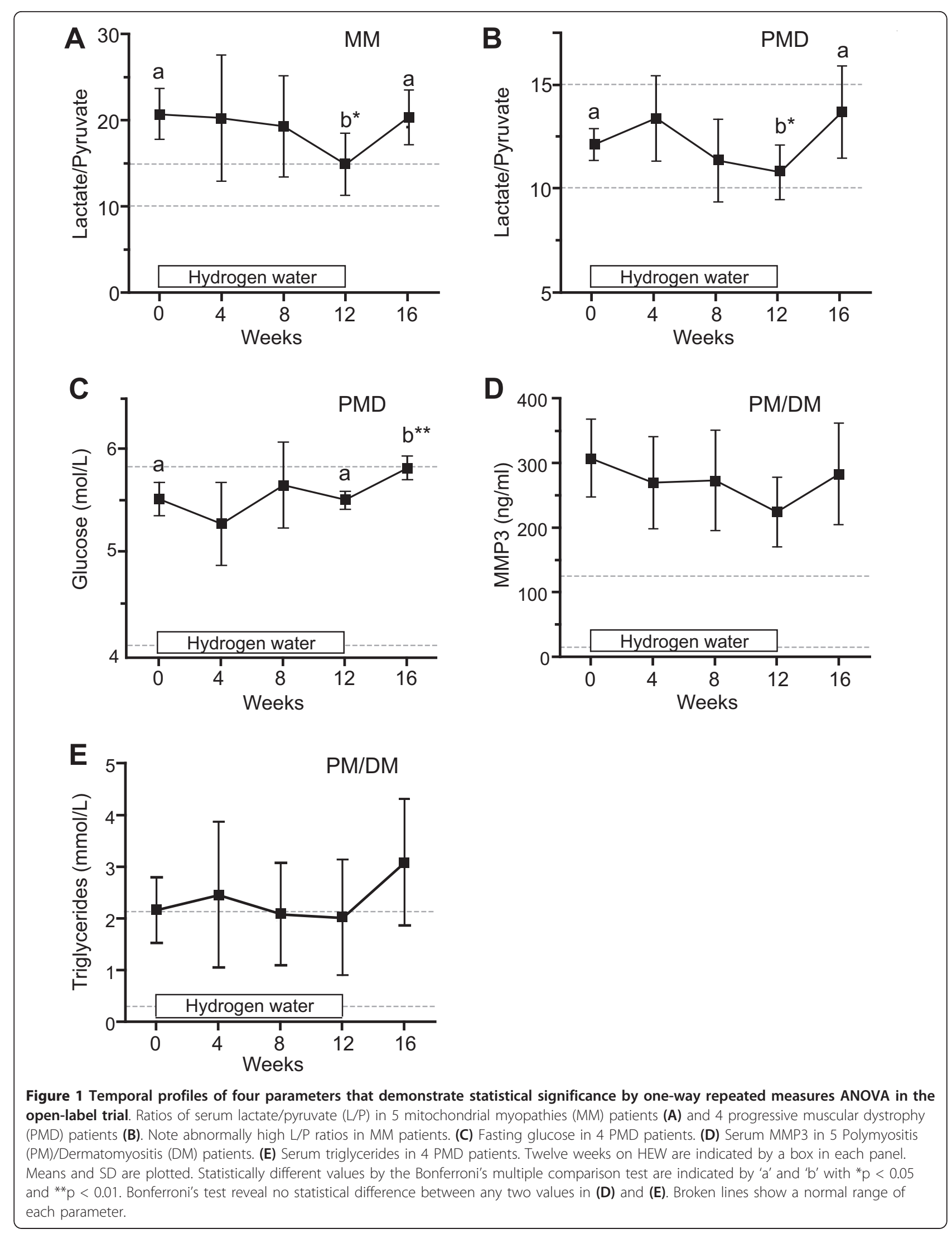


Table 2 Randomized, double-blind, placebo-controlled, crossover trial of HEW in 10 DM and 12 MM patients

\begin{tabular}{|c|c|c|c|c|c|c|c|c|}
\hline & \multicolumn{4}{|c|}{ Dermatomyositis (DM) } & \multicolumn{4}{|c|}{ Mitochondrial myopathies (MM) } \\
\hline & \multicolumn{2}{|c|}{ Hydrogen water } & \multicolumn{2}{|c|}{ Placebo water } & \multicolumn{2}{|c|}{ Hydrogen water } & \multicolumn{2}{|c|}{ Placebo water } \\
\hline & 0 week & 8 weeks & 0 week & 8 weeks & 0 week & 8 weeks & 0 week & 8 weeks \\
\hline CK (U/L) & $88.7 \pm 24.3$ & $106.1 \pm 88.2$ & $93.5 \pm 45.0$ & $99.5 \pm 86.7$ & $165 \pm 86.6$ & $120 \pm 55.5$ & $142.0 \pm 69.4$ & $221 \pm 235$ \\
\hline HbA1c (\%) & $6.23 \pm 1.28$ & $6.34 \pm 1.55$ & $6.27 \pm 1.44$ & $6.16 \pm 1.28$ & $6.09 \pm 0.94$ & $6.12 \pm 1.05$ & $6.06 \pm 1.22$ & $6.06 \pm 1.02$ \\
\hline Fasting glucose $(\mathrm{mmol} / \mathrm{L})$ & $8.27 \pm 3.62$ & $7.81 \pm 2.91$ & $6.70 \pm 2.11$ & $6.48 \pm 1.90$ & $6.05 \pm 1.43$ & $5.67 \pm 1.99$ & $6.02 \pm 1.46$ & $6.11 \pm 1.69$ \\
\hline Lactate (mmol/L) & $1.93 \pm 0.78$ & $1.81 \pm 0.87$ & $1.80 \pm 0.89$ & $1.65 \pm 0.77$ & $1.76 \pm 0.67^{*}$ & $1.61 \pm 0.48^{*}$ & $1.49 \pm 0.49^{*}$ & $1.70 \pm 0.57^{*}$ \\
\hline L/P ratio & $13.1 \pm 6.0$ & $11.5 \pm 2.6$ & $12.1 \pm 2.88$ & $15.2 \pm 8.3$ & $18.7 \pm 8.8$ & $17.9 \pm 7.7$ & $7.12 \pm 13.4$ & $17.7 \pm 8.6$ \\
\hline Creatinine $(\mu \mathrm{mol} / \mathrm{L})$ & $59.1 \pm 15.6$ & $59.1 \pm 13.6$ & $58.3 \pm 15.0$ & $59.0 \pm 19.7$ & $53.6 \pm 18.3$ & $52.0 \pm 17.4$ & $54.8 \pm 20.3$ & $57.5 \pm 22.3$ \\
\hline BUN (mmol/L) & $5.36 \pm 1.48$ & $4.82 \pm 1.57$ & $5.78 \pm 1.71$ & $4.86 \pm 1.56$ & $6.08 \pm 2.09$ & $5.39 \pm 1.54$ & $6.24 \pm 1.46$ & $6.34 \pm 2.76$ \\
\hline Uric Acid ( $\mu \mathrm{mol} / \mathrm{dL})$ & $303 \pm 88$ & $320 \pm 68$ & $313 \pm 75$ & $321 \pm 83$ & $413 \pm 316$ & $375 \pm 229$ & $447 \pm 387$ & $408 \pm 284$ \\
\hline T-chol (mmol/L) & $5.23 \pm 0.78$ & $5.15 \pm 0.85$ & $5.21 \pm 0.61$ & $4.95 \pm 0.92$ & $4.61 \pm 0.78$ & $4.80 \pm 0.57$ & $4.69 \pm 0.78$ & $4.68 \pm 0.71$ \\
\hline LDL-chol (mmol/L) & $3.06 \pm 0.82$ & $2.93 \pm 0.75$ & $2.97 \pm 0.80$ & $3.03 \pm 0.84$ & $2.69 \pm 0.68$ & $2.82 \pm 0.58$ & $2.73 \pm 0.67$ & $2.79 \pm 0.60$ \\
\hline HDL-chol (mmol/L) & $1.59 \pm 0.48$ & $1.56 \pm 0.36$ & $1.53 \pm 0.47$ & $1.43 \pm 0.48$ & $1.47 \pm 0.04$ & $1.55 \pm 0.34$ & $1.52 \pm 0.34$ & $1.45 \pm 0.35$ \\
\hline Triglycerides (mmol/L) & $1.54 \pm 0.65$ & $1.83 \pm 0.76$ & $1.86 \pm 0.80$ & $1.85 \pm 0.48$ & $1.16 \pm 0.58$ & $0.92 \pm 0.35$ & $0.97 \pm 0.36$ & $1.02 \pm 0.49$ \\
\hline WBC $\left(10^{9} / \mathrm{L}\right)$ & $11.7 \pm 6.4$ & $10.9 \pm 3.1$ & $9.66 \pm 2.68$ & $11.2 \pm 4.7$ & $5.5 \pm 0.1$ & $5.7 \pm 1.8$ & $5.81 \pm 1.78$ & $5.8 \pm 1.9$ \\
\hline $\mathrm{RBC}\left(10^{12} / \mathrm{L}\right)$ & $4.28 \pm 0.05$ & $4.32 \pm 0.35$ & $4.37 \pm 0.44$ & $4.35 \pm 0.44$ & $4.15 \pm 0.04$ & $4.17 \pm 0.61$ & $4.26 \pm 0.67$ & $4.15 \pm 0.56$ \\
\hline Platelets $\left(10^{9} / \mathrm{L}\right)$ & $271 \pm 10$ & $302 \pm 69$ & $306 \pm 54$ & $312 \pm 79$ & $203 \pm 40$ & $214 \pm 44$ & $216 \pm 48$ & $225 \pm 50$ \\
\hline Hematocrit (\%) & $0.380 \pm 0.004$ & $0.384 \pm 0.040$ & $0.392 \pm 0.052$ & $0.391 \pm 0.047$ & $0.373 \pm 0.004$ & $0.378 \pm 0.060$ & $0.386 \pm 0.063$ & $0.376 \pm 0.058$ \\
\hline MMP3 (ng/ml) & $245 \pm 122$ & $232 \pm 84.1$ & $217 \pm 93.5$ & $221 \pm 112$ & n.d. & n.d. & n.d. & n.d. \\
\hline $\operatorname{lgG}(\mathrm{mg} / \mathrm{dl})$ & $1211 \pm 357$ & $1244 \pm 305$ & $1202 \pm 340$ & $1282 \pm 353$ & n.d. & n.d. & n.d. & n.d. \\
\hline
\end{tabular}

Values represent mean \pm SD. n.d., not determined. ${ }^{*} \mathrm{p}<0.05$ by two-way repeated measures ANOVA.

mitochondrial inner membranes and increases production of reactive oxygen species (ROS), which further damages mETS $[13,14]$. Reduction of the $\mathrm{L} / \mathrm{P}$ ratios in the open-label and double-blind studies suggests that hydrogen alleviates mETS dysfunction in MM either by scavenging ROS or by yet unidentified signaling mechanisms.

MMP3 belongs to a family of calcium-dependent zinc proteinases induced by cytokines and secreted by inflammatory cells. MMPs enhance T-cell migration and adhesion, and also degrade the extracellular matrix proteins [15]. MMP3 is increased in a fraction of DM patients [16]. MMP3 may facilitate lymphocyte adhesion and enhance T-cell-mediated cytotoxicity by degrading extracellular matrix proteins in DM. Hydrogen improved serum MMP3 levels in the open-label and double-blind studies, which is expected to ameliorate pathogenic inflammatory processes that culminates in muscle fiber destruction.

We observed less prominent effects with the doubleblind study compared to the open-label study. The lack of statistically significance in the double-blind study is possibly due to a lower amount of HEW (1.0 vs. 0.5 liter per day) and to a shorter observation period (12 vs. 8 weeks). In the open-label study, drinking 1.0 liter of HEW was not readily accommodated by most myopathic patients. Hydrogen does not show simple dose- response relationship in rodents [2-4], and ad libitum administration of even 5\%-saturated HEW markedly attenuates development of Parkinson's disease in mice [17]. We thus reduced the amount of hydrogen to 0.5 liter in the double-blind trial, and also shortened the observation period to minimize the burden on the participants. This, however, might have masked effects of HEW. Indeed, when we compare studies of diabetes mellitus type 2 [6], the current open-label trial, and metabolic syndrome [9], the participants took 0.9, 1.0, and 1.5-2.0 liters of HEW, respectively. Ratios of total cholesterol/HDL-cholesterol are available at 8 weeks in all the studies, and are changed to $103.8 \%, 98.6 \%$, and $95.8 \%$, respectively, of those before hydrogen administration, which is in accordance with a dose-response effect of HEW. Additionally, among the two previous studies $[6,9]$ and the current open-label and double-blind studies, the most prominent effects are observed with 1.52.0 liters of HEW. As drinking a large amount of HEW is not easily accommodated by most patients especially in winter, a threshold effect and/or a dose-response effect should be further elaborated for each pathological state.

\section{Conclusions}

HEW is effective for mitochondrial dysfunction in MM and inflammatory processes in DM. Hydrogen may have 


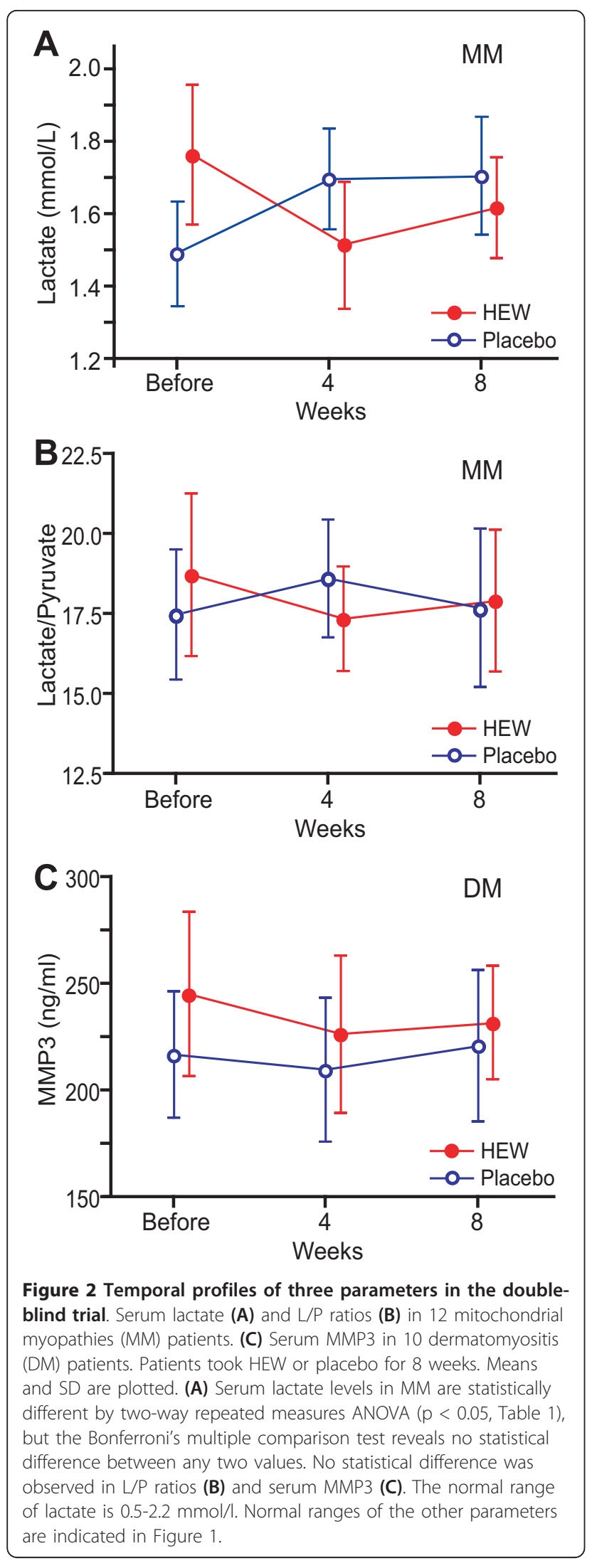

a threshold effect or a dose-response effect and 1.0 liter or more per day of HEW is likely to be required to exert beneficial effects.

\section{Abbreviations}

HEW: hydrogen-enriched water; PMD: progressive muscular dystrophy; PM: polymyositis; DM: dermatomyositis; MM: mitochondrial myopathies; CPEO: chronic progressive external ophthalmoplegia; MELAS: mitochondrial myopathy with lactic acidosis and stroke-like episodes; MMP3: matrix metalloproteinase-3.

\section{Acknowledgements}

We would like to thank the patients for their participation in these studies. We thank Fumiko Ozawa for her technical assistance. This work was supported by Grants-in-Aid from the Ministry of Health, Labor, and Welfare of Japan and the Ministry of Education, Culture, Sports, Science, and Technology of Japan.

\section{Author details}

'Division of Neurogenetics, Center for Neurological Diseases and Cancer, Nagoya University Graduate School of Medicine, 65 Tsurumai, Showa-ku, Nagoya 466-8550, Japan. ${ }^{2}$ Faculty of Pathophysiology and Therapeutics, Aichi Medical University College of Nursing, 21 Karimata Yazako, Nagakute-cho, Aichi-gun, Aichi 480-1195, Japan. ${ }^{3}$ Department of Neurology, Aichi Medical University School of Medicine, 21 Karimata Yazako, Nagakute-cho, Aichi-gun, Aichi 480-1195, Japan. ${ }^{4}$ Department of Biomedical Sciences, College of Life and Health Sciences, Chubu University, 1200 Matsumoto, Kasugai, Aichi 4878501, Japan. ${ }^{5}$ Department of Longevity and Aging Research, Gifu International Institute of Biotechnology, 1-1 Nakafudogaoka, Kakamigahara, Gifu 504-0838, Japan.

\section{Authors' contributions}

$\mathrm{Tl}$ and $\mathrm{KS}$ examined patients and acquired data. $\mathrm{Ml}^{1}$ and $\mathrm{Tl}$ organized data and performed statistical analysis. $\mathrm{Ml}^{1}$ and $\mathrm{KO}$ wrote the paper. $\mathrm{Ml}^{4}, \mathrm{Ml}^{5}$, and $\mathrm{KO}$ conceived the study. All authors read and approved the final manuscript.

\section{Competing interests}

The authors declare that they have no competing interests.

Received: 24 June 2011 Accepted: 3 October 2011

Published: 3 October 2011

\section{References}

1. Ohsawa I, Ishikawa M, Takahashi K, Watanabe M, Nishimaki K, Yamagata K, Katsura K, Katayama Y, Asoh S, Ohta S: Hydrogen acts as a therapeutic antioxidant by selectively reducing cytotoxic oxygen radicals. Nat Med 2007, 13:688-694.

2. Ohta S, Nakao A, Ohno K: The 2011 Medical Molecular Hydrogen Symposium: An Inaugural Symposium of the Journal Medical Gas Research. Med Gas Res 2011, 1:10.

3. Nakao A, Sugimoto R, Billiar TR, McCurry KR: Therapeutic Antioxidant Medical Gas. J Clin Biochem Nutr 2009, 44:1-13.

4. Hong $\mathrm{Y}$, Chen $\mathrm{S}$, Zhang JM: Hydrogen as a selective antioxidant: a review of clinical and experimental studies. J Int Med Res 2010, 38:1893-1903.

5. Itoh T, Fujita Y, Ito M, Masuda A, Ohno K, Ichihara M, Kojima T, Nozawa Y, Ito M: Molecular hydrogen suppresses FcepsilonRI-mediated signal transduction and prevents degranulation of mast cells. Biochem Biophys Res Commun 2009, 389:651-656.

6. Kajiyama S, Hasegawa G, Asano M, Hosoda H, Fukui M, Nakamura N, Kitawaki J, Imai S, Nakano K, Ohta M, Adachi T, Obayashi H, Yoshikawa T: Supplementation of hydrogen-rich water improves lipid and glucose metabolism in patients with type 2 diabetes or impaired glucose tolerance. Nutr Res 2008, 28:137-143.

7. Nakayama M, Kabayama S, Nakano H, Zhu WJ, Terawaki H, Nakayama K, Katoh K, Satoh T, Ito S: Biological Effects of Electrolyzed Water in Hemodialysis. Nephron Clin Pract 2009, 112:C9-C15.

8. Nakayama M, Nakano H, Hamada H, Itami N, Nakazawa R, Ito S: A novel bioactive haemodialysis system using dissolved dihydrogen $(\mathrm{H}-2)$ 
produced by water electrolysis: a clinical trial. Nephrol Dial Transplant 2010, 25:3026-3033.

9. Nakao A, Toyoda Y, Sharma P, Evans M, Guthrie N: Effectiveness of Hydrogen Rich Water on Antioxidant Status of Subjects with Potential Metabolic Syndrome-An Open Label Pilot Study. J Clin Biochem Nutr 2010, 46:140-149.

10. Kang K-M, Kang Y-N, Choi I-B, Gu Y, Kawamura T, Toyoda Y, Nakao A: Effects of drinking hydrogen-rich water on the quality of life of patients treated with radiotherapy for liver tumors. Med Gas Res 2011, 1:11.

11. Ono H, Nishijima Y, Adachi1 N, Tachibana S, Chitoku S, Mukaihara S, Sakamoto M, Kudo Y, Nakazawa J, Kaneko K, Nawashiro H: Improved brain MRI indices in the acute brain stem infarct sites treated with hydroxyl radical scavengers, Edaravone and hydrogen, as compared to Edaravone alone. A non-controlled study. Med Gas Res 2011, 1:12.

12. DiMauro S: Pathogenesis and treatment of mitochondrial myopathies: recent advances. Acta Myologica 2010, 29:333-338.

13. Wei YH, Lu CY, Wei CY, Ma YS, Lee HC: Oxidative stress in human aging and mitochondrial disease-consequences of defective mitochondrial respiration and impaired antioxidant enzyme system. Chin J Physiol 2001, 44:1-11.

14. McKenzie M, Liolitsa D, Hanna MG: Mitochondrial disease: mutations and mechanisms. Neurochem Res 2004, 29:589-600.

15. Sternlicht MD, Werb Z: How matrix metalloproteinases regulate cell behavior. Annu Rev Cell Dev Biol 2001, 17:463-516.

16. Nishijima C, Hayakawa I, Matsushita T, Komura K, Hasegawa M, Takehara K, Sato S: Autoantibody against matrix metalloproteinase-3 in patients with systemic sclerosis. Clin Exp Immunol 2004, 138:357-363.

17. Fujita K, Seike T, Yutsudo N, Ohno M, Yamada H, Yamaguchi H, Sakumi K, Yamakawa Y, Kido MA, Takaki A, Katafuchi T, Nakabeppu Y, Noda M: Hydrogen in drinking water reduces dopaminergic neuronal loss in the 1-methyl-4-phenyl-1,2,3,6-tetrahydropyridine mouse model of Parkinson's disease. PLoS One 2009, 4:e7247.

doi:10.1186/2045-9912-1-24

Cite this article as: Ito et al.: Open-label trial and randomized, doubleblind, placebo-controlled, crossover trial of hydrogen-enriched water for mitochondrial and inflammatory myopathies. Medical Gas Research 2011 1:24.

\section{Submit your next manuscript to BioMed Central and take full advantage of:}

- Convenient online submission

- Thorough peer review

- No space constraints or color figure charges

- Immediate publication on acceptance

- Inclusion in PubMed, CAS, Scopus and Google Scholar

- Research which is freely available for redistribution

Submit your manuscript at www.biomedcentral.com/submit 